\title{
Combination of Raman tweezers and quantitative differential interference contrast microscopy for measurement of dynamics and heterogeneity during the germination of individual bacterial spores
}

\author{
Pengfei Zhang \\ Lingbo Kong \\ Guiwen Wang \\ East Carolina University \\ Department of Physics \\ Greenville, North Carolina 27858-4353
}

\section{Peter Setlow}

University of Connecticut Health Center

Department of Molecular, Microbial, and Structural Biology

Farmington, Connecticut 06030-33052

Yong-qing Li

East Carolina University

Department of Physics

Greenville, North Carolina 27858-4353

\begin{abstract}
Raman tweezers and quantitative differential interference contrast (DIC) microscopy are combined to monitor the dynamic germination of individual bacterial spores of Bacillus species, as well as the heterogeneity in this process. The DIC bias phase is set properly such that the brightness of DIC images of individual spores is proportional to the dipicolinic acid (DPA) level of the spores, and an algorithm is developed to retrieve the phase image of an individual spore from its DIC image. We find that during germination, the rapid drop in both the intensity of the original DIC image and the intensity of the reconstructed phase image precisely corresponds to the release of all DPA from that spore. The summed pixel intensity of the DIC image of individual spores adhered on a microscope coverslip is not sensitive to the drift of the slide in both horizontal and vertical directions, which facilitates observation of the germination of thousands of individual spores for long periods of time. A motorized stage and synchronized image acquisition system is further developed to effectively expand the field of view of the DIC imaging. This quantitative DIC technique is used to track the germination of hundreds or thousands of individual spores simultaneously. @ 2010 Society of Photo-Optical Instrumentation Engineers. [DOI: 10.1117/1.3494567]
\end{abstract}

Keywords: Raman tweezers; quantitative differential interference contrast microscopy; spore germination; germination heterogeneity.

Paper 10277R received May 24, 2010; revised manuscript received Jul. 29, 2010; accepted for publication Aug. 17, 2010; published online Sep. 30, 2010.

\section{Introduction}

Bacterial spores of Bacillus species are metabolically dormant, extremely resistant to a variety of harsh conditions, and can survive for years in this state. ${ }^{1}$ However, spores can rapidly return to life through germination followed by outgrowth if appropriate nutrients and some non-nutrient agents are provided. $^{2}$ One important step in spore germination is the rapid release of the spore's large depot $(\sim 10 \%$ of spore dry wt) of pyridine-2, 6-dicarboxylic acid [dipicolinic acid (DPA)] and its associated divalent cations, predominantly $\mathrm{Ca}^{2+}$ (CaDPA), that is present in the dormant spores' central region or core. ${ }^{2}$ Germination of spores in populations exhibits significant heterogeneity, with some spores germinating very rapidly and some extremely slowly, as is indicated by the variable time between the addition of germinants and the release of CaDPA. ${ }^{3-5}$ Noninvasive monitoring of hundreds or thousands of individual spores under physiological germination conditions will thus be necessary to quantify the heterogeneity in the germination of individual spores in populations.

Address all correspondence to Yong-qing Li, East Carolina University, Department of Physics, Greenville, North Carolina 27858-4353; Tel: 252-328-1858; Fax: 252-328-6314; E-mail: liy@ecu.edu
Phase contrast $(\mathrm{PC})^{6}$ and differential interference contrast $(\mathrm{DIC})^{7}$ microscopy are well-known techniques for imaging transparent phase objects without the need for exogenous contrast agents, which produce very little intensity contrast in bright-field microscopy. Although different principles and optical components are used, both of these methods convert an invisible phase image into an amplitude image. The optical phase shifts experienced by the illumination light that passes through transparent objects are associated with the specimen's refractive index and contain both structural and dynamic features. Therefore, the changes in intensity of PC or DIC images that result from variations in phase shift allow real-time inspection of biological specimens noninvasively. Dormant bacterial spores are transparent and appear phase-bright in PC and DIC microscopy due to the high refractive index in the spore's core that results from the high levels of CaDPA and the core's relatively low water content. However, when spores germinate, CaDPA is released into the environment and the spore's peptidoglycan cortex is hydrolyzed such that the spore core's refractive index decreases, thus appearing phase-dark. PC and DIC microscopy has been used to monitor the kinetics of germination of single Bacillus and Clostridium spores. ${ }^{8-11}$

1083-3668/2010/15(5)/056010/9/\$25.00 @ 2010 SPIE 
However, because the relationship between the intensity of images and the phase of objects is generally nonlinear, only qualitative information has been obtained by PC and DIC techniques.

Phase-shifted differential interference contrast (PS-DIC) microscopy was developed to obtain the linear phase gradient of an object along the shear direction through a mathematical operation on four frames of a phase-shifted image. ${ }^{12}$ Furthermore, when a spiral phase integration (SPI) technique is applied to a PS-DIC image, linear phase images can be retrieved. ${ }^{13,14}$ However, this quantitative phase technique requires multiple frames to produce a single PS-DIC image, and also requires the rotation of the direction of shear to apply the SPI algorithm, ${ }^{13,15}$ which makes it difficult to use for real-time detection of kinetic processes in biological specimens. In recent years, several quantitative phase microscopy techniques have been developed that are based on the interference between the spatially varying field through the sample and a uniform reference beam, including Fourier phase microscopy (FPM) ${ }^{16}$ Hilbert phase microscopy (HPM), ${ }^{17,18}$ and diffraction phase microscopy (DPM). ${ }^{19}$ These techniques have been used for monitoring cell growth ${ }^{20}$ and membrane fluctuations of red blood cells. ${ }^{19}$

Raman spectroscopy is another useful technique in biochemical studies that benefits from this spectroscopy's high sensitivity and rapid response to subtle changes in molecular structure. ${ }^{21-23}$ When Raman spectroscopy is combined with confocal microscopy and optical trapping, the resultant Raman tweezers (LTRS) have the ability to probe biochemical processes at the single-cell level in liquid media, ${ }^{24,25}$ and have been used to monitor the kinetics of germination and heat inactivation of individual Bacillus spores. ${ }^{4,5,26}$ However, this technique becomes less efficient when hundreds of individual spores need to be monitored simultaneously. Recently, phase contrast microscopy was combined with LTRS, which allowed observation of the germination kinetics of hundreds of individual Bacillus cereus spores simultaneously. ${ }^{10}$ However, due to the artifacts of Zernike phase contrast, quantitative phase (refractive index) information on the spores was not retrieved. ${ }^{15}$ In addition, to combine with Raman tweezers spectroscopy, a special external phase configuration in the microscope has to be used. Furthermore, since the depth of focus is shallower in PC microscopy than in DIC microscopy, PC observation of spore germination requires higher stability of microscope stage in the vertical direction.

In this work, we report a system that combines Raman tweezers and quantitative DIC microscopy for studies of germination dynamics of individual bacterial spores. The advantages in choosing DIC microscopy are: 1. it has no artifacts of Zernike phase contrast such that quantitative phase images can be retrieved from the original DIC images; 2 . ordinary high numerical aperture (NA) objectives can be used for both DIC images and Raman spectroscopy and no special external phase design is needed; and 3. DIC images have higher contrast and wider depth of focus, lowering the requirement for the stability of the microscope stage. We first established the correspondence between CaDPA release using Raman spectroscopy and the changes in the intensity of DIC images of single spores using DIC imaging, and then applied quantitative DIC imaging to measure the heterogeneity in germination of hundreds of individual spores in a population. An image in which the intensity is linearly proportional to the optical phase of that spore was retrieved. During germination, the changes in the intensity of the reconstructed phase image have essentially the same correlation with the CaDPA release of that spore. To expand the full field of the DIC imaging, we used a motorized microscope stage and synchronously acquired the DIC images of each field of view. By this technique, we were able to simultaneously monitor thousands of individual spores during the germination process for up to $2 \mathrm{~h}$.

\section{Materials and Methods}

\subsection{Bacillus Species Used, Preparation, and Germination}

The Bacillus species used in this work were all wild-type strains and were Bacillus subtilis PS533, ${ }^{27}$ Bacillus cereus T (originally obtained from Halvorson), and Bacillus megaterium QM B1551 (originally obtained from Levinson). Spores of these species were prepared and stored as described. $^{28-30}$ All spores used in this work were free $(>98 \%)$ of growing or sporulating cells and germinated spores, as determined by phase contrast microscopy. Prior to germination, the spores were heat activated in water for $30 \mathrm{~min}$ at $70^{\circ} \mathrm{C}$ (B. subtilis), $20 \mathrm{~min}$ at $65^{\circ} \mathrm{C}$ (B. cereus $)$, or $15 \mathrm{~min}$ at $60^{\circ} \mathrm{C}$ (B. megaterium), and then put on ice for $>15$ min. The germination conditions used were as follows: B. subtilis, $37^{\circ} \mathrm{C}$ with various concentration of L-alanine in 25-mM Tris- $\mathrm{HCl}$ buffer ( $\mathrm{pH} 8.3) ;$ B. cereus, $30^{\circ} \mathrm{C}$ with $100-\mu \mathrm{M}$ L-alanine in $25-\mathrm{mM}$ Tris-HCl buffer ( $\mathrm{pH} 8.3$ ); and B. megaterium, $30^{\circ} \mathrm{C}$ with $1-\mathrm{mM}$ D-glucose in $25-\mathrm{mM}$ KPO4 buffer (pH 7.4).

\subsection{Laser Tweezers and Raman Spectroscopy}

The LTRS system used in this work was as described. ${ }^{10}$ As shown in Fig. 1(a), a diode laser at $780 \mathrm{~nm}$ was introduced into an inverted microscope (TE2000-S, Nikon Instruments, Lewisville, Texas) and formed a trap by the objective with high numerical aperture $(100 \times$, NA1.3, Nikon, Lewisville, Texas). Single spores were trapped at the focus of the objective, and the Raman scattering light from the trapped spore that was excited by the trapping laser was collected by the same objective. The collected backward Raman scattering light was recorded by a spectrometer (LS-785, Princeton Instruments, Trenton, New Jersey) equipped with a chargecoupled device (CCD) detector (PIXIS 400, Princeton Instruments, Trenton, New Jersey). The Raman spectra were recorded in the range from 600 to $1800 \mathrm{~cm}^{-1}$, with a resolution of about $6 \mathrm{~cm}^{-1}$. A background spectrum was also acquired under the same conditions without a spore in the trap and subtracted from the spectra of spores.

\subsection{Differential Interference Contrast Imaging and Phase Reconstruction}

As shown in Fig. 1(a), the DIC images of spores produced by the illumination of the halogen lamp source and the differential interference of transmitted light through the DIC module mounted on the microscope were captured with a 16-bit digital imaging CCD camera (QSI 520, Quantum Scientific Im- 

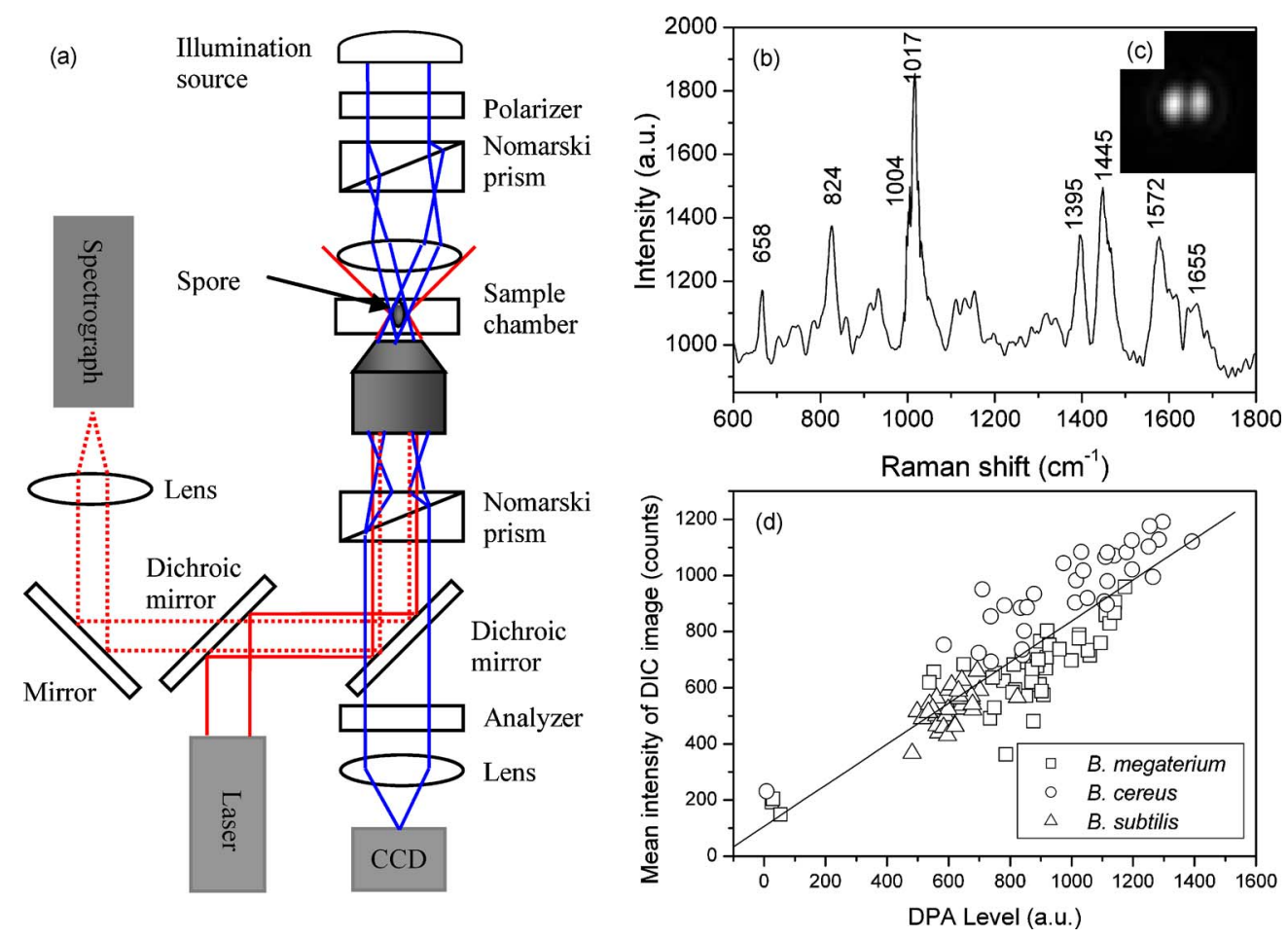

Fig. 1 Combination of Raman tweezers and DIC microscopy for the measurement of Bacillus spores. (a) Schematic diagram of the setup. (b) Raman spectrum of a typical dormant Bacillus spore trapped by laser tweezers. (c) DIC image of the trapped dormant spore. (d) Intensities of DIC images versus the CaDPA levels of trapped dormant spores as determined by Raman spectroscopy. 50 randomly chosen spores of $B$. cereus, $B$. subtilis, and B. megaterium were analyzed. The solid curve is a fitting line.

aging, Poplarville, Mississippi), or a 12-bit digital CCD camera (CCE-B013-U, Mightex Systems, Toronto, Canada). A background image was taken under the same conditions without a spore in the view field and subtracted from the DIC images of spores. The DIC module was set such that the axes of the polarizer and analyzer were crossed, and thus the bias phase $2 \theta$ between the two orthogonally polarized beams was $\pi,^{13} 2 \theta=\pi$. Under this configuration, the 2-D DIC image intensity produced was given by ${ }^{13,15}$

$$
\begin{aligned}
I(x, y)= & I_{1}(x, y)+I_{2}(x+\Delta x, y) \\
& -2\left[I_{1}(x, y) I_{2}(x+\Delta x, y)\right]^{1 / 2} \cos [\Delta \phi(x, y)],
\end{aligned}
$$

where $I_{1}$ and $I_{2}$ are the intensities of the two polarization components separated by a shear distance of $\Delta x$ set by the Nomarski prism, $\Delta \phi$ is the phase difference between the two polarization components that is induced by the spore, and the $x$ axis is assigned along the direction of the shear, which is determined by the Nomarski prisms in the DIC microscope. The optical phase $\phi$ is proportional to the index of refraction $n(x, y)$ of the spore and the traveling-path difference $\Delta l(x, y)$ of the illumination beams in the spore, $\phi(x, y)$ $=(2 \pi / \lambda) n(x, y) \Delta l(x, y)$, where $\lambda$ is the wavelength of the illumination lights. Assuming that the intensity of illumination light is uniform over the whole spore area, the intensities of two polarization components are the same, and the absorption of the spore is negligible, then Eq. (1) is simplified as

$$
I(x, y)=I_{0}\{1-\cos [\Delta \phi(x, y)]\},
$$

where $I_{0}=2 I_{1}=2 I_{2}$. The phase gradient at the spore's center is nearly zero, so a single spore appears as two bright parts with a dark line in the center, as suggested by Eq. (2) and as shown in Fig. 1. For a small shear distance $\Delta x$ that is less or comparable to the diffraction limit of microscope resolution, the phase gradient along the shear direction is generally not extreme, i.e., $|\Delta \phi(x, y)| \ll \pi$, and Eq. (2) is approximated as

$$
I(x, y)=I_{0} \Delta \phi(x, y)^{2} .
$$

We then can obtain the phase gradient along the $x$ direction,

$$
\Delta \phi(x, y)=\left\{\begin{array}{rl}
\sqrt{I(x, y) / I_{0}}, & \Delta \phi>0 \\
-\sqrt{I(x, y) / I_{0}}, & \Delta \phi<0
\end{array} .\right.
$$

It is generally difficult to determine the sign of $\Delta \phi$ for objects with complicated phase gradient distributions. However, for a single spore, the dark line that crosses the spore's center and perpendicular to the shear direction is the position with zero phase gradient, as shown in Fig. 1, in which the shear direction is along the $x$ direction in the image in the insert. Therefore, the phase gradients on the left and right sides of the dark line should have opposite signs. Generally, the shear distance is much smaller than the size of the spore, so the spore's phase distribution $\phi(x, y)$ then can be obtained by integrating the phase gradient along the $x$ direction, 


$$
\phi(x, y)=\int \frac{\Delta \phi(x, y)}{\Delta x} d x
$$

\subsection{Motorized Stage and Synchronized Image Acquisition}

To monitor up to thousands of individual spores in a germination experiment, we needed to expand the field of view of the DIC imaging, which was about $120 \times 90 \mu \mathrm{m}$ in our system. To do this, we used a precision motorized $x-y$ stage (MAX203B-NIK, ThorLab, Newton, New Jersey) and developed a control software to synchronously move the stage and acquire the DIC images. The control system allows moving the microscope stage to the first field of view of the spore sample $(120 \times 90 \mu \mathrm{m})$ with a precision of $1 \mu \mathrm{m}$, recording a DIC image, and then moving to a second field of view while recording its DIC image, and so on. In a run, six different fields of view were recorded within $18 \mathrm{sec}$. Then the stage was moved back to the first field of view to record the next set of DIC images, and this rotation was repeated. In this way, the germination of 2000 to 3000 individual spores can be recorded with a resolution time of $\sim 18 \mathrm{sec}$, and for up to $\sim 2 \mathrm{~h}$. The resolution time in our system was limited by the speed of storing large image data files onto a computer hard disk after each run of image acquisition. A fast camera data storage system would significantly reduce the resolution time of the whole system.

\section{Experimental Results}

Figure 1(b) shows a typical Raman spectrum from a trapped dormant $B$. subtilis spore. The bands at 658, 824, 1017, 1395, and $1572 \mathrm{~cm}^{-1}$ are from CaDPA, ${ }^{24,31}$ and the intensity of the predominant band at $1017 \mathrm{~cm}^{-1}$ has been used to determine the CaDPA level of individual Bacillus spores. ${ }^{24}$ The high refractive index in the spore's core due to the large depot of CaDPA and the core's low water content was then converted into an amplitude image under a DIC microscope and was recorded by a CCD camera. Figure 1(c) shows the DIC image of the spore analyzed in Fig. 1(b). This image appears as two separate bright parts with a narrow dark gap between them due to the small phase gradient near the spore's center. Intuitively, the spores with brighter DIC images have higher DPA levels. To confirm the relationship between the brightness of the DIC image and spore's DPA level, 50 randomly chosen dormant spores each of B. subtilis, B. cereus, and B. megaterium were trapped, and their DIC images and Raman spectra were recorded. The laser power used for the measurement was $5 \mathrm{~mW}$, and the integration times for DIC imaging and spectrum acquisition were 0.5 and $30 \mathrm{~s}$, respectively. The CaDPA level in individual spores was determined from the Raman scattering intensity of the band at $1017 \mathrm{~cm}^{-1}$ (averaged over five adjacent data points), as described previously. ${ }^{24}$ The mean intensity of the DIC image was calculated by averaging the counts of pixels over a region of $40 \times 40$ pixels (corresponding to $3 \times 3 \mu \mathrm{m}$ in the microscope specimen) that covered the whole spore (size of 1 to $2 \mu \mathrm{m}$ ), and was plotted as a function of its DPA level determined by the height of the $1017-\mathrm{cm}^{-1}$ Raman band, as shown in Fig. 1(d). As indicated by the fitting line, the intensity of the spore's DIC image is proportional to its CaDPA level, both for the spores from the same species and for spores from different species.

It would be useful to monitor the germination of a single spore using DIC microscopy and Raman spectroscopy simultaneously, and to examine the relationship between the intensity of the spore's DIC image and its CaDPA level during germination, since CaDPA release is a marker for spore germination and results in a decrease in the refractive index of a spore's core. Although previous work addressed the changes in the appearance of DIC images of B. subtilis spores after the completion of germination, ${ }^{11}$ quantitative and dynamic information has never been obtained previously from DIC microscopy. To measure germination kinetics of single spores adhered on a microscope coverslip, $20 \mu \mathrm{l}$ of a heat-activated spore suspension $\left(\sim 7 \times 10^{6}\right.$ spores $\left./ \mathrm{ml}\right)$ was placed on the surface of a coverslip. After $1 \mathrm{~h}$ at $4^{\circ} \mathrm{C}$ to allow the spores to adhere, the spore suspension was removed by a pipette, leaving a thin film of spores on the surface of the coverslip. After this film of spores was dried in a refrigerator, the coverslip was mounted onto a chamber on the microscope stage. The sample chamber was kept at $37^{\circ} \mathrm{C}\left(B\right.$. subtilis) or $30^{\circ} \mathrm{C}(B$. cereus and B. megaterium), and was sealed with a cover glass after adding the germinant solution preheated at the appropriate germination temperature. The microscope was adjusted to the center, the laser was focused on a randomly chosen spore, and then the Raman spectra and DIC images of that spore were recorded with exposure times of 5 and $0.5 \mathrm{~s}$, respectively. Only a $40 \times 40$ pixels area of the CCD camera (full resolution of $1600 \times 1200$ pixels) was used to record the DIC image of a single spore. To synchronize the acquisition of Raman spectra and DIC images, a visual basic program was developed to trigger the spectrographic CCD for Raman spectroscopy and the imaging CCD camera for DIC images at time intervals of 5 and $1 \mathrm{~s}$, respectively. In addition, for each coordinate $y$ of the DIC image, the position of minimum intensity of the original DIC image along the $x$ direction was located, the phase gradients in the left and right sides of this position were assigned opposite signs, and then the phase image of the spore was reconstructed through integration along the $x$ direction. Figure 2(a) shows the Raman spectra, original DIC images, and reconstructed phase images of a typical $B$. cereus spore adhered on a cover slip at several stages during its germination with $100-\mu \mathrm{M}$ L-alanine in $25-\mathrm{mM}$ Tris-HCl buffer $(\mathrm{pH} \mathrm{8.3)}$. As indicated, after 13 min of incubation, the spore contained the same amount of CaDPA and gave the same DIC images and phase images as when the measurement began. However, 0.5 min later, all CaDPA-related Raman bands had disappeared, and the intensities of the DIC and phase images were also greatly decreased. No obvious changes in the Raman spectra and only minor decreases in the DIC and phase images were observed over the next $3.5 \mathrm{~min}$. To quantitatively describe these changes, the averaged pixel intensities of the DIC and phase images as well as the CaDPA level of that spore were plotted as a function of incubation time in Fig. 2(b), where all of the intensities were normalized to their values at the time when the measurement began. The CaDPA level and the mean intensities of the DIC image and phase image were nearly unchanged until a time defined as $T_{\text {lag }}$, when all of these dropped rapidly in $\sim 0.5 \mathrm{~min}$. During this rapid CaDPA release, the level of CaDPA decreased to zero at a time defined as $T_{\text {release. }}$. However, the intensities of 


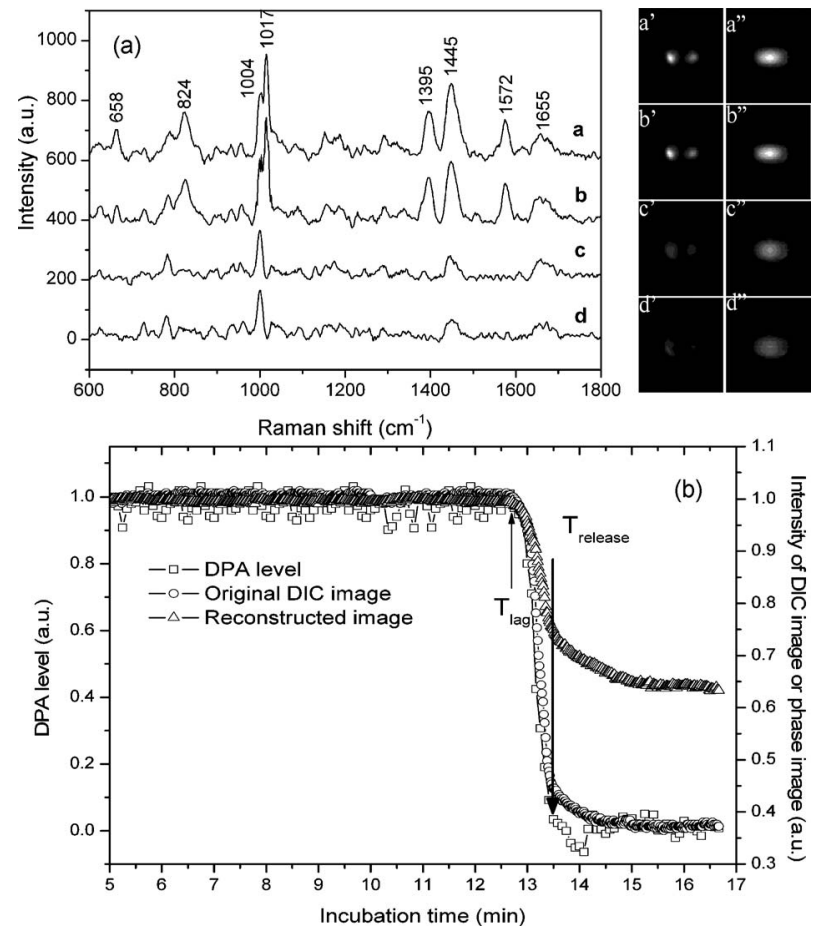

Fig. 2 Monitoring of a single $B$. cereus spore adhered on a microscope coverslip during germination with 100- $\mu \mathrm{M} \mathrm{L}$-alanine using Raman spectroscopy and quantitative DIC microscopy. (a) Raman spectra, DIC images, and reconstructed phase images of the spore at several stages during germination. Curves a through $d$ are for incubation times of $5,13,13.5$, and $16.5 \mathrm{~min}$, respectively. The baselines were shifted for display. Images to the right are DIC images (left colum) and phase images (right colum) of that spore taken at the corresponding times. (b) Intensity of Raman scattering at $1017 \mathrm{~cm}^{-1}$. The intensities of original DIC images and reconstructed phase images of the spore analyzed in (a) are expressed as a function of incubation time. All of these intensities are normalized to the corresponding values at the time when the measurement began.

the DIC image and phase image were deceased only 55 and $25 \%$, respectively, at $T_{\text {release }}$ followed by slow decreases to stable nonzero values. The slow decreases in the intensities of the DIC image and phase image after $T_{\text {release }}$ are due primarily to a decrease in the refractive index of the spore core because of the degradation of the spore's peptidoglycan cortex and the attendant swelling of the core due to significant water uptake. ${ }^{8,10}$ As indicated in Fig. 2, the kinetic parameters of $T_{\text {lag }}$ and $T_{\text {release }}$ during the spore's germination can be obtained from DIC microscopy as precisely as from Raman spectroscopy. Essentially identical results were obtained with other B. cereus spores and with spores of B. subtilis and $B$. megaterium (data not shown).

With this correspondence between the DIC image and the Raman spectrum in hand, it was then straightforward to simultaneously monitor the germination of large numbers of spores on a microscope coverslip, thus allowing use of DIC microscopy to study the heterogeneity in the germination of large numbers of individual spores in populations. To collect the kinetic parameters of hundreds or thousands of spores, a thin film of spores was prepared on the surface of a microscope coverslip and then mounted in a microscope sample chamber, as described before. After preheated germinant so-

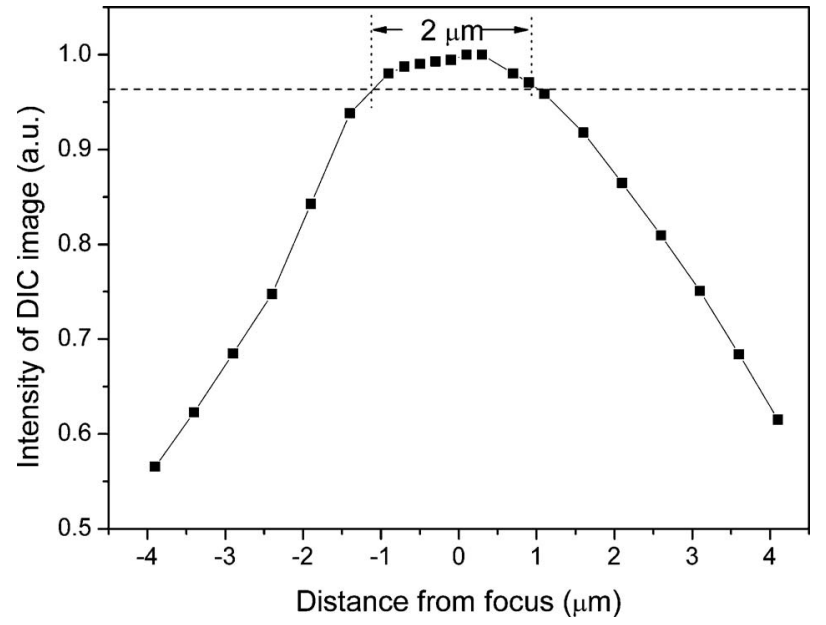

Fig. 3 Mean DIC image intensity of a typical B. subtilis spore adhered on a microscope coverslip versus the distance of the spore away from the focus. The intensity of the DIC image was calculated by averaging the pixel counts over an area of $40 \times 40$ pixels $(3 \times 3 \mu \mathrm{m})$ that covered the whole spore.

lutions were added to the temperature-stabilized chamber, an appropriate field of view was chosen, brought into focus, and DIC images were recorded by a 16-bit CCD camera with an exposure time of $0.5 \mathrm{~s}$ and at a frame rate of $15 \mathrm{~s}$. These images were processed with a program in Matlab (Natick, Massachusetts) to locate each individual spore and to calculate the averaged pixel intensity of a $40 \times 40$ pixels region $(3 \times 3 \mu \mathrm{m})$ that was large enough to cover the whole individual spore (size of 1 to $2 \mu \mathrm{m}$ ). The full resolution of the CCD camera is $1600 \times 1200$ pixels, so in an ideal case, one experiment can follow the germination of $\sim 1200$ spores. In practice, each field of view can record 300 to 500 spores, depending on the concentration of spore suspension as well as the distribution uniformity of spores on the coverslip. In a few cases, the cross-correlation coefficients between the first image and successive frames were calculated and used to correct any position drifts of the chamber along the horizontal direction. Besides the drift in the horizontal direction, the drift of the sample chamber in the vertical direction is also a potential problem for long observation periods. To address this concern, a dormant spore was adhered on a coverslip, and its DIC images at various vertical positions were recorded by changing the voltage applied to a microscope-objective piezo element (Mipos 100, Piezosystem jena, Hopedale, Massachusetts) that was attached to the objective. The averaged pixel intensity of a $40 \times 40$ pixels region was calculated and plotted as a function of the distance from the focus in Fig. 3. The mean intensity of the DIC image decreased by $<3 \%$ when the spore was moved out of focus within a range from -1 to $1 \mu \mathrm{m}$, a range that is easily controlled manually or by feedback devices.

Figure 4 shows the DIC images of germinating B. subtilis spores adhered on a microscope coverslip. The images were recorded at $0,5,10$, and $30 \mathrm{~min}$ after the addition of mixture of 10-mM L-alanine and 25-mM Tris- $\mathrm{HCl}$ buffer $(\mathrm{pH} 8.3)$. For illustration, only small portions of $320 \times 320$ pixels clipped from the full resolution DIC images are shown. Indi- 

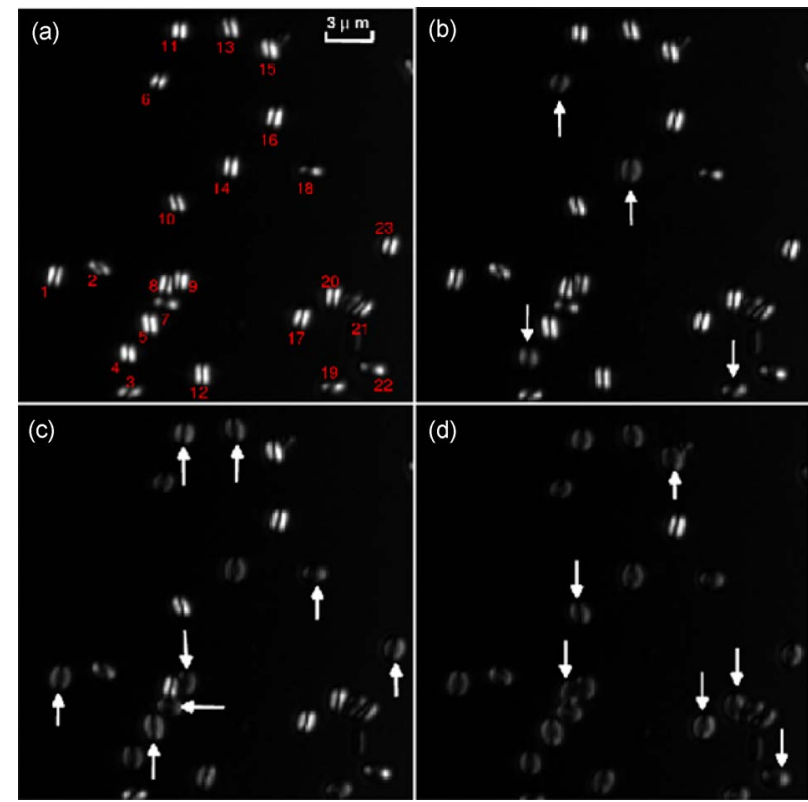

Fig. 4 DIC images of germinating B. subtilis spores adhered on a microscope coverslip. The images were recorded 0, 5, 10, and $30 \mathrm{~min}$ after the addition of 10-mM L-alanine in 25-mM Tris-HCl buffer $(\mathrm{pH}$ 8.3); arrows mark the germinated spores at each time point. Only small portions of $320 \times 320$ pixels clipped from the full view of the DIC images are shown. The scale bar is $3 \mu \mathrm{m}$, and all images are at the same scale. All spores are numbered such that their germination can be tracked.
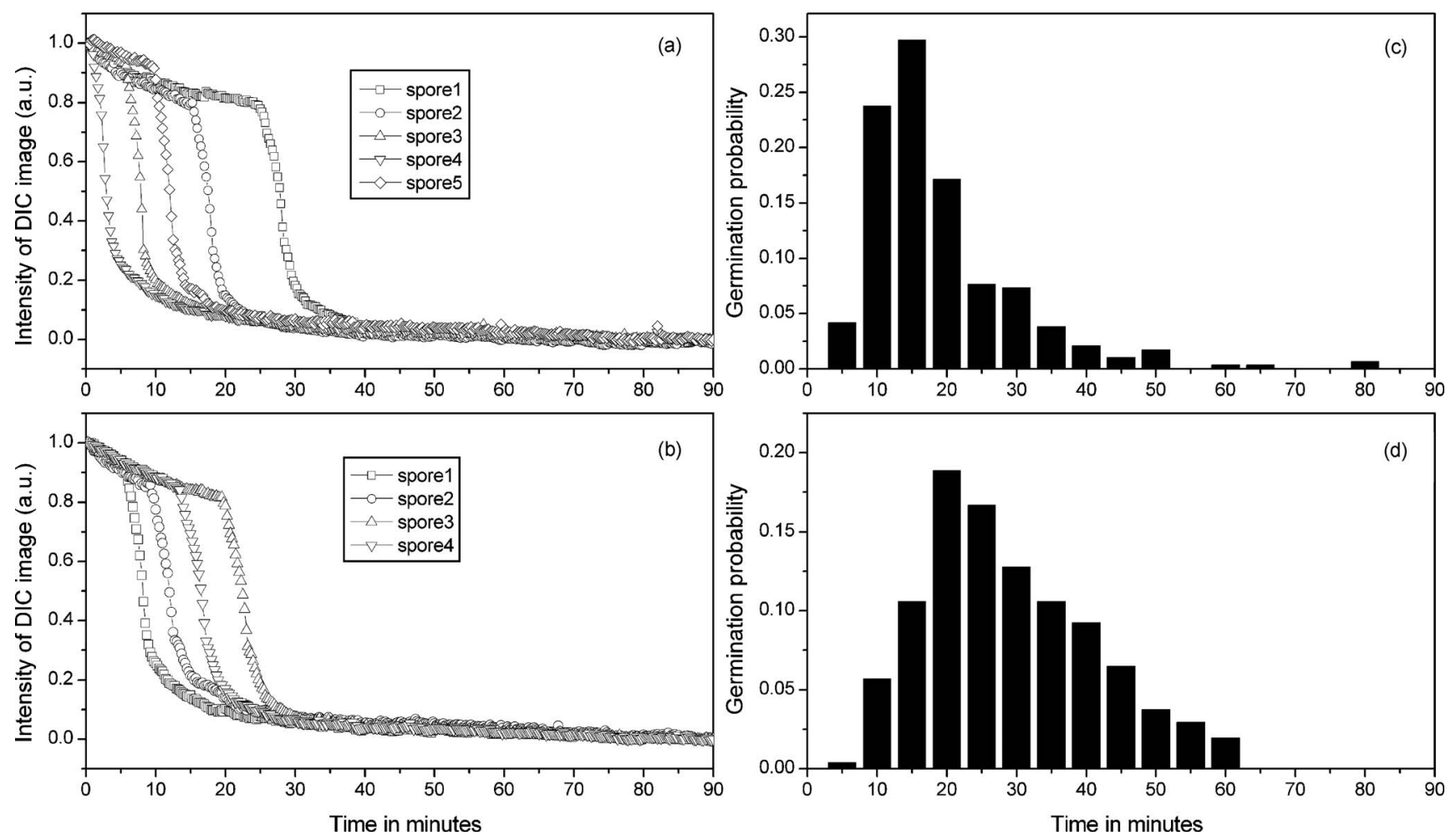

Fig. 5 L-Alanine germination of multiple B. subtilis spores adhered on a microscope slide. (a) DIC image intensities of five randomly chosen $B$. subtilis spores adhered on a microscope slide during germination with $10-\mathrm{mM} \mathrm{L}$-alanine and $25-\mathrm{mM}$ Tris- $\mathrm{HCl}$ buffer (pH 8.3). (b) DIC intensity of four adjacent spores from the same experiment as in (a) that were separated by less than $6 \mu \mathrm{m}$. For purposes of display, the DIC intensity values after 90 min of incubation were subtracted from the intensities of DIC images at various times, and the values were normalized to the values at the time germinants were added. (c) and (d) are the distribution probabilities of $T_{\text {release }}$ for spores germinated with $10-\mathrm{mM}$ and $50-\mu \mathrm{M} \mathrm{L-alanine,}$ respectively. 
germination kinetics of four adjacent spores separated by less than $6 \mu \mathrm{m}$ from each other in the experiment described in Fig. 4 were studied, and their DIC image intensities are shown in Fig. 5(b) as a function of incubation time. It is obvious that although these spores almost certainly experienced the same local environment during their germination, they exhibited different germination kinetics, as revealed by their different $T_{\text {lag }}$ and $T_{\text {release }}$ times. This finding indicates that minor differences in the local germination conditions were almost certainly not a major factor causing the heterogeneity in germination of individual spores in populations.

Both $T_{\text {lag }}$ and $T_{\text {release }}$ are important kinetic parameters that can be used to characterize the heterogeneity in spore germination, since the rapid release of spore's CaDPA is the first easily measured event during germination. However, in some case, i.e., in spores that lack the cortex lytic enzyme CwlJ in which the rate of release of CaDPA during germination is much slower than with wild-type spores, ${ }^{5} T_{\text {release }}$ is a time point that can be more easily defined. To quantify the heterogeneity between the germination of individual spores, it is necessary to collect the $T_{\text {release }}$ times for hundreds or thousands of spores. Figure 5(c) shows the distribution probability of $T_{\text {release }}$ from $291 \mathrm{~B}$. subtilis spores germinated with $10-\mathrm{mM}$ L-alanine (a saturating germinant concentration) in $25-\mathrm{mM}$ Tris- $\mathrm{HCl}$ buffer $(\mathrm{pH} 8.3)$. The $T_{\text {release }}$ times were distributed over a very wide time range with a maximum probability located at around $15 \mathrm{~min}$. When the concentration of L-alanine was decreased to a subsaturating concentration of $50 \mu \mathrm{M}$, the time with the maximum germination probability was shifted to $20 \mathrm{~min}$, and the distribution width of $T_{\text {release }}$ was also increased, as shown in Fig. 5(d), where 509 spores were analyzed. We also used a motorized stage with high accuracy and high reproducibility (see Sec. 2) to record the DIC images of 2000 to 3000 spores germinating with an even lower L-alanine concentration, where the very low germination efficiency $(<10 \%)$ required high data acquisition efficiency (data not shown).

\section{Discussion}

In this work, we set the bias phase $2 \theta$ of the DIC microscope at $\pi$, such that the DIC images of spores had the maximum contrast, although these images did not directly show the actual sizes and shapes of the spores. With this setting, the intensity of the DIC image was approximately proportional to the square of the phase gradient of that spore along the direction of shear. When a simple algorithm was applied, a phase image in which the intensity is linearly proportional to the spore's optical phase was retrieved from the original DIC image. Since the change in the optical phase is related to the specific molecular events during the dynamic germination process of single spores, quantitative DIC imaging is capable of determining the important parameters of the spore's germination with high temporal resolution and high sensitivity, when combined with Raman spectroscopy and laser tweezers.

The results reported in this work demonstrated several advantages of the combination of quantitative DIC imaging and Raman spectroscopy for the observation of individual spores and their germination in physiological conditions. The first advantage is that the intensity of the original DIC image of an individual dormant spore was proportional to that spore's
CaDPA level. This conclusion was drawn from trapped spores that had their long axes perpendicular to the focal plane, and thus the phase gradients along any radial direction were nearly the same, assuming that the spore's CaDPA and other components were distributed symmetrically along the radical direction. This conclusion might not be appropriate for spores adhered on a microscope coverslip on which spores are oriented randomly, with different orientations resulting in different DIC intensity. The orientation of a spore affects its optical phase gradient along the shear axis of polarization and thus affects the brightness of its DIC image [see Eq. (2)]. As shown in Fig. 4, the vertically orientated spores (e.g., spores 11 and 12) appeared as two parallel bright bars, and the horizontally orientated spores (e.g., spores 7 and 18) appeared as two bright spots with greater separation and less summed pixel intensity. However, this does not prevent the application of DIC microscopy for the observation of germination of individual spores on a coverslip, since kinetic parameters of germinantion can still be determined from the relative changes in the intensity of the DIC image. This is also the reason why phase reconstruction is necessary to determine the absolute phase of the spore during germination.

The second advantage of the combined system was to show the precise correspondence of the rapid drop in the intensities of the DIC images and the phase images to the CaDPA release of that spore. Apparently, during the germination of single spores, the changes in the DIC image and the phase image were highly correlated with changes in Raman scattering at CaDPA-specific bands. This correspondence makes it possible to monitor the germination kinetics of hundreds of individual spores by quantitative DIC microscopy alone, without the need for Raman spectroscopy that generally can only be used to monitor the germination of a single spore.

The third advantage was that large numbers of individual spores can be monitored simultaneously in an experiment. When a motorized stage is used, the DIC images of thousands of spores can be recorded rapidly, and these images can be used to study the germination parameters of spores with very low germination probability. In general, the number of detectable spores and the time resolution of image acquisition in our system were limited by the speed of storing large image data files onto a computer hard disk. This limitation could be overcome by saving the image data onto a RAM disk or using a high speed computer to calculate the DIC intensities in the memory while acquiring the images and then discarding the image data. With these modifications, it would then be possible to monitor up to $\sim 10^{4}$ germinating spores simultaneously with a time resolution of the order of $10 \mathrm{sec}$ by using DIC microscopy and a motorized stage. In addition, since the slow decreases in the intensities of the DIC image and the reconstructed phase image are related to the events following CaDPA release believed to be associated with the degradation of the spore's cortex, ${ }^{8}$ the kinetic parameters related to these events also can be obtained from quantitative DIC microscopy.

The fourth advantage is that the computed pixel intensity of a single spore's DIC image over the whole cell region $(3 \times 3 \mu \mathrm{m})$ is not sensitive to the drifts of the microscope stage or the sample chamber in either the horizontal or vertical direction. The tolerance of $+/-1 \mu \mathrm{m}$ for the drift in the 
vertical direction lowers the requirement for the focus-locking system and allows observation of spore germination over long time periods.

When quantitative DIC in combination with Raman spectroscopy was used to measure the dynamics during the germination of Bacillus spores, several results were observed. The germination of individual spores in a population is highly heterogeneous, with some spores germinating very rapidly and some extreme slowly, although the time required for the release of the majority of a spore's CaDPA was nearly constant. In addition, this heterogeneity is not the result of different microscopic physical or chemical environments during the germination. The results in this work further indicated that germination of spores with lower germinant concentrations exhibited higher heterogeneity than spores germinating with high concentrations. Recent work has indicated that heat activation and levels of nutrient germinant receptors also affect $T_{\text {release }}$ times, and has suggested that the numbers of functional or activated nutrient germinant receptors per spore are the major factor causing heterogeneity in spore germination. $^{32,33}$

In summary, we develop a combined system of Raman tweezers and quantitative DIC microscopy. This system allows the recording of time-lapse Raman spectra as well as DIC images of single bacterial spores either in an optical trap or adhered on a coverslip during nutrient-triggered germination. The phase image of individual germinating spores can be retrieved from the recorded DIC images. A motorized stage and a synchronized imaging acquisition system allow simultaneously monitoring of the germination of 2000 to 3000 individual spores for $\sim 2 \mathrm{~h}$. The temporal resolution for DIC or Raman data acquisition from a single spore is $5 \mathrm{sec}$, and the temporal resolution for acquisition of multiple full-field DIC images is $18 \mathrm{sec}$. This quantitative DIC microscopy has already proven to be a useful tool for the real-time observation of the germination of hundreds or thousands of individual spores in a population, and this technique should also be suitable for the measurement of heterogeneity in spore inactivation by moist heat. ${ }^{26}$

\section{Acknowledgments}

This work was supported by a Multi-University Research Initiative (MURI) award from the Department of Defense (PS/ YQL), and by a grant from the Army Research Office (YQL/ $\mathrm{PS})$. We are grateful to Jing $\mathrm{Yu}$ for participation in some experiments.

\section{References}

1. P. Setlow, "Spores of Bacillus subtilis: their resistance to and killing by radiation, heat and chemicals," J. Appl. Microbiol. 101, 514-525 (2006).

2. P. Setlow, "Spore germination," Curr. Opin. Microbiol. 6, 550-556 (2003).

3. D. Chen, S. S. Huang, and Y. Q. Li, "Real-time detection of kinetic germination and heterogeneity of single Bacillus spores by laser tweezers Raman spectroscopy," Anal. Chem. 78, 6936-6941 (2006).

4. L. Peng, D. Chen, P. Setlow, and Y. Q. Li, "Elastic and inelastic light scattering from single bacterial spores in an optical trap allows the monitoring of spore germination dynamics," Anal. Chem. 81, 40354042 (2009).

5. B. Setlow, L. Peng, C. A. Loshon, Y.-Q. Li, G. Christie, and P. Setlow, "Characterization of the germination of Bacillus megaterium spores lacking enzymes that degrade the spore cortex," J. Appl. Microbiol. 107, 318-328 (2009).

6. F. Zernike, "How I discovered phase contrast," Science 121, 345-349 (1955).

7. G. Nomarski and A. R. Weill, "Application à la métallographie des methods interférentielles à denux ondes polarisées," Rev. Metall. 2 , 121-128 (1955).

8. T. Hashimoto, W. R. Frieben, and S. F. Conti, "Germination of single bacterial spores," J. Bacteriol. 98, 1011-1020 (1969).

9. C. M. P. Billon, C. J. McKirgan, P. J. McClure, and C. Adair, "The effect of temperature on the germination of single spores of Clostridium botulinum," J. Appl. Microbiol. 82, 48-56 (1997).

10. L. Kong, P. F. Zhang, P. Setlow, and Y. Q. Li, "Characterization of bacterial spore germination using integrated phase contrast microscopy Raman spectroscopy and optical tweezers," Anal. Chem. 82, 3840-3847 (2010).

11. R. G. K. Leuschner, D. P. Ferdinando, and P. J. Lillford, "Structural analysis of spores of Bacillus subtilis during germination and outgrowth," Colloids Surf., B 19, 31-41 (2000).

12. P. Hariharan and M. Roy, "Achromatic phase-shifting for two wavelength phase-stepping interferometery," Opt. Commun. 126, 220-222 (1994).

13. M. R. Arnison, K. G. Larkin, C. J. R. Sheppard, N. I. Smith, and C. J. Cogswell, "Linear phase imaging using differential interference contrast microscopy," J. Microsc. 214, 7-12 (2004).

14. S. V. King and C. J. Cogswell, "A phase-shifting DIC technique for measuring 3D phase objects: experimental verification," Proc. SPIE 5324, 191-196 (2004).

15. S. V. King, A. Libertun, R. Piestun, C. J. Cogswell, and C. Preza, "Quantitative phase microscopy through differential interference imaging," J. Biomed. Opt. 13, 024020 (2008).

16. G. Popescu, L. P. Deflores, J. C. Vaughan, K. Badizadegan, H. Iwai, R. R. Dasari, and M. S. Feld, "Fourier phase microscopy for investigation of biological structures and dynamics," Opt. Lett. 29, 25032505 (2004).

17. G. Popescu, T. Ikeda, C. A. Best, K. Badizadegan, R. R. Dasari, and M. S. Feld, "Erythrocyte structure and dynamics quantified by Hilbert phase microscopy," J. Biomed. Opt. 10, 060503 (2005).

18. T. Ikeda, G. Popescu, R. R. Dasari, and M. S. Feld, "Hilbert phase microscopy for investigating fast dynamics in transparent systems," Opt. Lett. 30, 1165-1167 (2005).

19. G. Popescu, Y. K. Park, W. Choi, R. R. Dasari, M. S. Feld, and K. Badizadegan, "Imaging red blood cell dynamics by quantitative phase microscopy," Blood Cells Mol. Dis. 41, 10-16 (2008).

20. G. Popescu, Y. K. Park, N. Lue, C. Best-Popescu, L. Deflores, R. R. Dasari, M. S. Feld, and K. Badizadegan, "Optical imaging of cell mass and growth dynamics," Am. J. Physiol.: Cell Physiol. 295, 538544 (2008).

21. G. J. Puppels, H. S. P. Garritsen, G. M. J. Segersnolten, F. F. M Demul, and J. Greve, "Raman microspectroscopic approach to the study of human granulocytes," Biophys. J. 60, 1046-1056 (1991).

22. A. R. Mahadevan-Jansen and R. Richards-Kortum, "Raman spectroscopy for the detection of cancers and precancers," J. Biomed. Opt. 1, 31-70 (1996).

23. T. C. Bakker Schut, G. J. Puppels, Y. M. Kraan, J. Greve, L. L. van der Maas, and C. G. Figdor, "Intracellular carotenoid levels measured by Raman microspectrocopy: comparison of lymphocytes from lung cancer patients and healthy individuals," Int. J. Cancer 74, 20-25 (1997).

24. S. S. Huang, D. Chen, P. L. Pelczar, V. R. Vepachedu, P. Setlow, and Y. Q. Li, "Levels of $\mathrm{Ca}^{2+}$-dipicolinic acid in individual Bacillus spores determined using microfluidic Raman tweezers," J. Bacteriol. 189, 4681-4687 (2007).

25. C. G. Xie, M. A. Dinno, and Y. Q. Li, "Near-infrared Raman spectroscopy of single optically trapped biological cells," Opt. Lett. 27, 249-251 (2002)

26. P. F. Zhang, L. B. Kong, P. Setlow, and Y. Q. Li, "Characterization of wet heat inactivation of single spores of Bacillus species by dual-trap Raman spectroscopy and elastic light scattering," Appl. Environ. Microbiol. 76, 1796-1805 (2010).

27. B. Setlow and P. Setlow, "Role of DNA repair in Bacillus subtilis spore resistance," J. Bacteriol. 178, 3486-3495 (1996).

28. M. O. Clements and A. Moir, "Role of the gerI operon of Bacillus cereus 569 in the response of spores to germinants," J. Bacteriol. 180, 1787-1797 (1998). 
Zhang et al.: Combination of Raman tweezers and quantitative differential interference contrast microscopy...

29. S. Goldrick and P. Setlow, "Expression of a Bacillus megaterium sporulation-specific gene in Bacillus subtilis," J. Bacteriol. 155 1459-1462 (1983).

30. M. Paidhungat, B. Setlow, A. Driks, and P. Setlow, "Characterization of spores of Bacillus subtilis which lack dipicolinic acid," J. Bacteriol. 182, 5505-5512 (2000).

31. A. Magge, A. C. Granger, P. G. Wahome, B. Setlow, V. R. Vepachedu, C. A. Loshon, L. Peng, D. Chen, Y. Q. Li, and P. Setlow,
"Role of dipicolinic acid in the germination, stability, and viability of spores of Bacillus subtilis," J. Bacteriol. 190, 4798-4807 (2008).

32. P. F. Zhang, W. Garner, X. Yi, Ji Yu, Y. Q. Li, and P. Setlow, "Factors affecting the variability in the time between addition of nutrient germinants and rapid DPA release during germination of spores of $\mathrm{Ba}$ cillus species," J. Bacteriol. (In press).

33. X. Yi and P. Setlow, "Studies of the commitment step in the germination of spores of Bacillus species," J. Bacteriol. (In press). 